\title{
Revisited definition of GLE
}

\author{
Stepan Poluianov* \\ Space Climate Research Unit, University of Oulu, Finland, \\ Sodankylä Geophysical Observatory, University of Oulu, Finland, E-mail: \\ stepan.poluianov@oulu.fi.
}

\section{Ilya Usoskin}

Space Climate Research Unit, University of Oulu, Finland,

Sodankylä Geophysical Observatory, University of Oulu, Finland.

\section{Alexander Mishev}

Space Climate Research Unit, University of Oulu, Finland.

\section{Don Smart}

SSSRC, Nashua, NH, USA.

\section{Margaret Shea}

SSSRC, Nashua, NH, USA.

The worldwide network of neutron monitors (NMs) includes two stations, South Pole (SOPO/B) and Dome C (DOMC/B), which are exceptionally sensitive to solar energetic particles. Their locations on the high Antarctic plateau make them favorable for detection of low-energy particles because of the low geomagnetic rigidity cutoff and the thin atmosphere above. This pair of cosmic ray stations is able to register relatively weak solar energetic particle events, which would not have been detected by the NM network otherwise. Since DOMC/B station is in operation only since 2015, now the NM network is more sensitive to a SEP event than ever before. Considering such weak events as the "official" ground-level enhancements (GLEs) may break the homogeneity of the GLE definition and cause an observational bias in studies based on the GLE occurrence rate over decades.

In order to keep the "official" GLE list homogeneous, we propose to slightly modify the conventional definition of a GLE, which refers to a statistically significant enhancement of the count rate of at least two differently located neutron monitors over the background. The proposed change is as follows: "... at least two neutron monitors, one of which is located near the sea level". We also propose an introduction of a new class of SEP events called sub-GLE to identify the events registered by high-elevation polar NMs but not satisfying the revised definition of a GLE.

35th International Cosmic Ray Conference - ICRC2017

10-20 July, 2017

Bexco, Busan, Korea

\footnotetext{
* Speaker.
} 


\section{Introduction}

Solar energetic particles (SEP) are particles (mostly protons) sporadically accelerated by the Sun to the energies of the order of several $\mathrm{MeV}$, sometimes exceeding a hundred $\mathrm{MeV}$. During rare strong SEP events, some particles can be accelerated to the energy of up to a few GeV. On the way to the Earth's atmosphere, SEP are partly shielded by the geomagnetic field and those, which can pass it in polar regions, are mostly absorbed in the atmosphere. However, particles with the energy above several hundreds $\mathrm{MeV}$ can initiate sufficiently intensive atmospheric cascades of reactions, products of which can be registered on the ground.

Historically, first SEP events were observed by ground-based instruments as an enhancement of the count rate over the galactic cosmic ray background and later called "ground level enhancements" or "ground level events" (GLE) [e.g., 4, and references therein]. Although space-borne instruments can measure SEP flux directly, its high-energy component during strong SEP events is studied by GLE events observed with the global network of ground-based neutron monitors (NM). This network is developed in several directions including start of operation of new high-elevation polar neutron monitors with enhanced sensitivity to low-energy cosmic ray particles. This causes a need in revision of the present definition of a GLE event in order to keep the existing GLE database as homogeneous as possible, as discussed below.

\section{High-elevation polar neutron monitors}

The location plays a crucial role in cosmic ray measurements performed with neutron monitors. In the atmosphere, the average cosmic ray cascade is attenuated at altitudes below approximately 15-20 km above the sea level [e.g., 2]. Therefore, elevation of a neutron monitor increases the count rate and reduces the lower energy limit for detectable cosmic ray particles. Another important location factor is the geomagnetic latitude of an instrument because it defines the geomagnetic cutoff effect, which can be roughly represented as the lower magnetic rigidity limit for cosmic ray particles able to reach a given location. The geomagnetic cutoff rigidity varies from zero to about $17 \mathrm{GV}$ over the globe (between the polar and equatorial regions, respectively) [e.g., 6 , and references therein].

For detection of relatively low-energy SEP (of the order of $\mathrm{MeV}$ and up to $\mathrm{GeV}$ ), both atmospheric and geomagnetic cutoffs are important, and the optimal location on the ground for that purpose would be a high-elevation polar site. There are two such places at the Earth: the top of the glacier at $3205 \mathrm{~m}$ a.s.l. in Greenland (the Northern hemisphere) and the Antarctic plateau with the average elevation of about $3000 \mathrm{~m}$ a.s.l. (the Southern hemisphere).

Unfortunately, there is no high-elevation cosmic ray station in Greenland, but there are two NM stations on the Antarctic plateau - the NMs at Amundsen-Scott South Pole station (SOPO/B) [3] and at Concordia station, Dome C (DOMC/B) [5] (Figure 1). Both cosmic ray stations have "standard" and "bare" (lead-free) NMs. SOPO/B are in work since 1964 (the "bare" part of NM since the end of 1986), DOMC/B started its operation in 2015. These stations can detect a relatively weak SEP event which would not be registered by other neutron monitors.

On 29 October 2015 there was a solar energetic particle (SEP) event, which caused an enhancement in the count rate only of SOPO/B and DOMC/B NMs and no response in other NMs of 


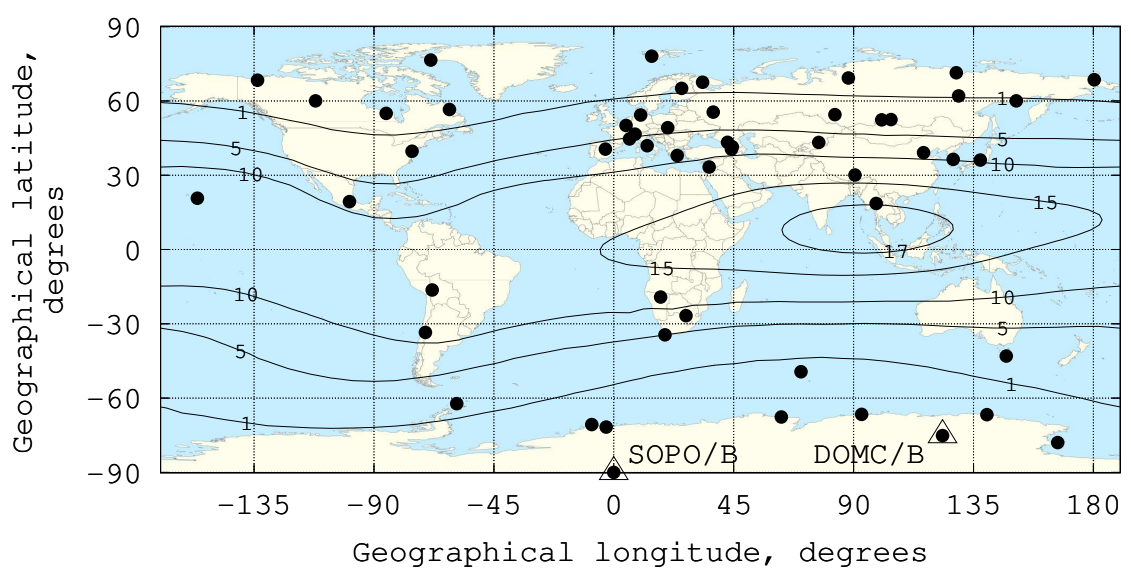

Figure 1: Map of neutron monitors. Triangles denote the South Pole and Dome $\mathrm{C}$ neutron monitors (labeled as $\mathrm{SOPO} / \mathrm{B}$ and $\mathrm{DOMC} / \mathrm{B}$, respectively). Contours with numbers indicate the geomagnetic cutoff rigidities (in GV).

the worldwide network (Figure 2). This can formally be considered as a new GLE, and we discuss it in the next section.
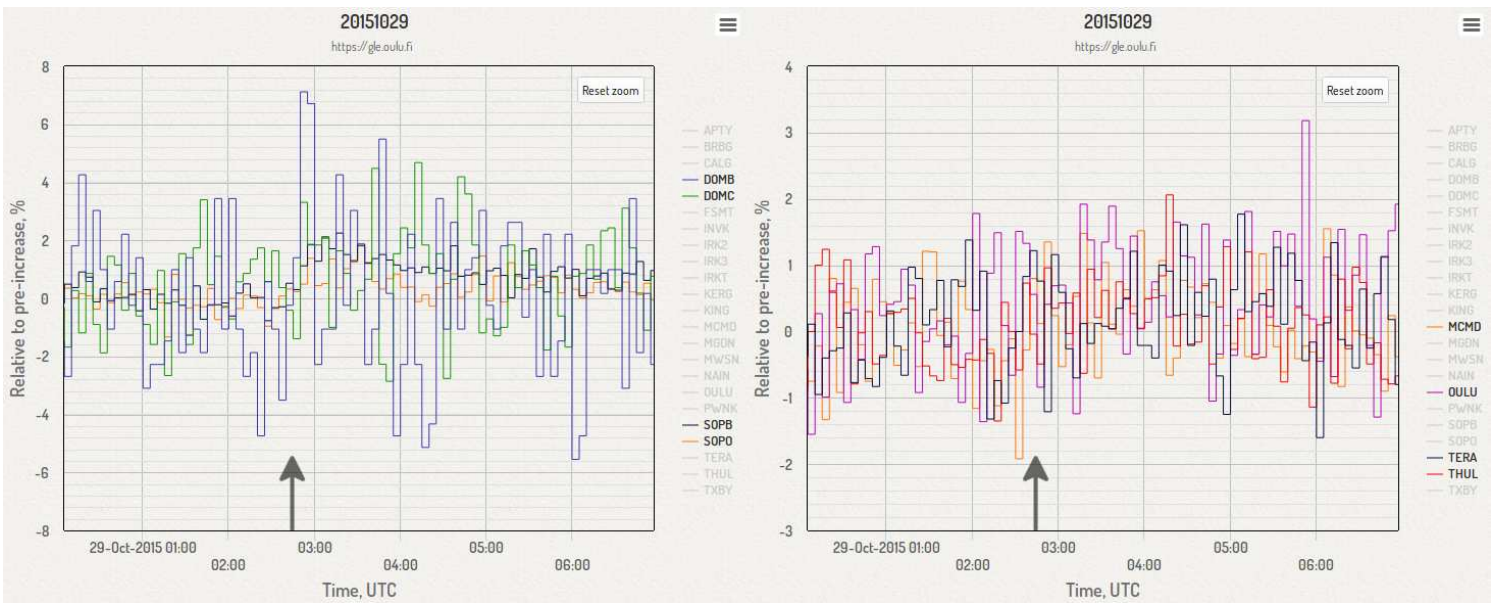

Figure 2: Solar energetic particle event on 29 October 2015 registered by SOPO/B and DOMC/B neutron monitors (the left panel). The right panel shows examples of data from other neutron monitors (namely, MCMD, OULU, TERA and THUL) with no response on the event. The vertical arrows indicate the beginning of the event. Data and plots from the GLE database gle. oulu. fi.

\section{Definitions of GLE and sub-GLE events}

The GLE is an enhancement in the count rate of a neutron monitor over the background formed by relatively slowly varying galactic cosmic rays. This phenomenon is caused by an SEP event hitting the Earth. Not every SEP event can cause a GLE, it should have particles that are sufficiently energetic to overcome the atmospheric and geomagnetic thresholds and be registered by a groundbased instrument. 
So far, 71 "official" GLEs have been registered since 1942 (according to the GLE database gle.oulu.fi), as acknowledged by the cosmic ray community. For identification of a GLE, a formal definition has been formulated by the community in the early 1970s:

A GLE event is registered when there are simultaneous statistically significant enhancements of the count rates of at least two differently located NMs and a corresponding enhancement in the proton flux measured by a space-borne instrument( $(s)$.

Before 2015 the global NM network had only one high-elevation polar NM station South Pole (SOPO/B), and thus, all the GLEs were registered by NMs including at least one sea-level instrument. With the installation of DOMC/B NM in 2015, the pair of highly sensitive to SEP instruments SOPO/B and DOMC/B will likely register more GLEs than it would have been done earlier. It has been confirmed by the SEP event on 29 October 2015 mentioned in the previous section (Figure 2). This fact distorts the homogeneity of the "official" list of GLEs and affects studies of SEP events based on the GLE occurance rates. In order to keep the list as homogeneous as possible, we propose to slightly modify the definition of GLE mentioned above with the following way (changes are highlighted with the bold font):

A GLE event is registered when there are simultaneous statistically significant enhancements of the count rates of at least two differently located NMs including at least one NM near the sea level and a corresponding enhancement in the proton flux measured by a space-borne instrument(s).

"Near the sea level" means not higher than one interaction length of the nucleonic component of the cosmic ray induced cascade above the sea level (viz. approximately $900 \mathrm{~g} / \mathrm{cm}^{2}$ or $1000 \mathrm{~m}$ a.s.l.). The new definition does not affect the present list of GLEs because, as was mentioned above, all the events were detected by NMs including at least one near the sea level.

According to the proposed definition, the event on 29 October 2015 registered only by highelevation polar NMs cannot be considered as a GLE, although it is a sufficiently strong SEP event that can be studied with neutron monitors. Because of that, we also propose a new class called "sub-GLE" for classification of such events expecting that more of them will be probably detected in the future [1]. The corresponding definition is proposed in this form:

A sub-GLE event is registered when there are simultaneous statistically significant enhancements of the count rates of at least two differently located high-elevation NMs and a corresponding enhancement in the proton flux measured by a space-borne instrument( $(s)$, but no statistically significant enhancement in the count rates of NMs near the sea level.

Those sub-GLEs can be listed in another part of the same database along with "standard" GLEs.

\section{Summary}

With installation of the high-elevation polar NM DOMC/B in addition to SOPO/B, the global NM network got a pair of NMs, which are very sensitive to low-energy cosmic rays. This is 
especially important for detection of GLE and potentially leads to more efficient registration of such events than before.

In order to keep the homogeneity of the list of GLEs, we propose to slightly modify the GLE definition with the additional condition that a GLE should be detected by at least one NM located near the sea level. Also we propose a new class "sub-GLE" for SEP events, which are detected only by high-elevation neutron monitors with reduced atmospheric cutoff and do not satisfy the revised definition of GLE.

\section{References}

[1] W. Atwell, A. J. Tylka, W. Dietrich, K. Rojdev, and C. Matzkind. Sub-GLE Solar Particle Events and the Implications for Lightly-Shielded Systems Flown During an Era of Low Solar Activity. In Int. Conf. Environ. Sys., Lunar and Planetary Science Conference Proceedings, 2015.

[2] G. A. Bazilevskaya, I. G. Usoskin, E. O. Flückiger, R. G. Harrison, L. Desorgher, R. Bütikofer, M. B. Krainev, V. S. Makhmutov, Y. I. Stozhkov, A. K. Svirzhevskaya, N. S. Svirzhevsky, and G. A. Kovaltsov. Cosmic ray induced ion production in the atmosphere. Space Sci. Rev., 137:149-173, 2008.

[3] P. Evenson, J. Bieber, J. Clem, and R. Pyle. South Pole Neutron Monitor Lives Again. International Cosmic Ray Conference, 11:459, 2011.

[4] L. Miroshnichenko. Solar cosmic rays. Springer International Publishing, Switzerland, 2015.

[5] S. Poluianov, I. Usoskin, M. Mishev, H. Moraal, H. Krüger, G. Casasanta, R. Traversi, and R. Udisti. Mini Neutron Monitors at Concordia Research Station, Central Antarctica. Journal of Astronomy and Space Sciences, 32:281-287, 2015.

[6] D.F. Smart and M.A. Shea. Fifty years of progress in geomagnetic cutoff rigidity determinations. Adv. Space Res., 44(10):1107-1123, 2009. 